\title{
An integrated circuit to null standby using energy provided by MEMS sensors
}

\author{
Roberto La Rosa ${ }^{1}$, A. Y. S. Pandiyan², Carlo Trigona ${ }^{3}$, Bruno Andòn ${ }^{3}$ Salvatore Baglio ${ }^{3}$ \\ ${ }^{1}$ STMicroelectronics, Italy \\ 2 Department of Electrical and Electronics Engineering, Imperial College London, UK \\ ${ }^{3}$ Department of Electrical, Electronics and Computer Engineering, University of Catania, Italy
}

\begin{abstract}
The advent of smart measurement systems and innovative wireless sensor networks evinces the necessity to develop novel solutions for conditioning circuits to be used in autonomous or quasi-autonomous measurement systems and sensing nodes. The main problem these systems still face is the question of how to supply the nodes in a cost-effective way, considering that, very often, a battery is required, and consequently, the maintenance labor and cost to replace or recharge it may be high. The main target is to increase battery lifetime by decreasing unnecessary energy consumption as much as possible. In this context, several solutions, including energy harvesters, have already been proposed. One of the main solutions is the reduction of power consumption by the measurement device while in standby, which, in most cases, represents a significant amount of the total power dissipation. To this end, authors have already addressed a zero-energy standby solution able to supply the power requested by the measurement equipment only when the appliance is turned on. In this paper, we present an integrated circuit solution suitable to be used with MEMS scale transducers. The validation and the characterisation of the system will be shown to demonstrate the suitability of the proposed method.
\end{abstract}

\section{Section: RESEARCH PAPER}

Keywords: MEMS; zero-energy standby; power management; wireless sensor networks; lithium ion battery; wireless sensor networks; Internet of Things

Citation: Roberto La Rosa, A. Y. S. Pandiyan, Carlo Trigona, Bruno Andò, Salvatore Baglio, An integrated circuit to null standby using energy provided by MEMS sensors, Acta IMEKO, vol. 9, no. 4, article 19, December 2020, identifier: IMEKO-ACTA-09 (2020)-04-19

Section Editor: Francesco Bonavolonta, University of Naples Federico II, Italy

Received October 31, 2019; In final form June 23, 2020; Published December 2020

Copyright: This is an open-access article distributed under the terms of the Creative Commons Attribution 3.0 License, which permits unrestricted use, distribution, and reproduction in any medium, provided the original author and source are credited.

Corresponding author: Roberto La Rosa: roberto.larosa@st.com; Carlo Trigona: carlo.trigona@dieei.unict.it

\section{INTRODUCTION}

In response to the advent of emerging technology such as IoT, Industry 4.0 and smart solutions in electronics and measurement, an interesting development has recently appeared regarding ultra-low-power solutions, self-powered methods, energy harvesting and novel conditioning circuits for sensors and transducers [1]-[4].

The common goal is to realise a sensing node having the prerogative to absorb small amounts of electrical power. These kinds of circuits are prominent in several applications, such as biomedical [5], industrial [6], smart solutions such as laptops, smartphones and gaming devices [7] and intelligent systems and novel measurement architectures such as smart homes, smart cities, etc. [8].

Along with reducing the size of electronic components and sensors (MEMS), manufacturers also focus on producing devices that consume less power. It is worth noting that battery life must be maximised, since replacement poses a real problem in node maintenance. In fact, this task is often very costly and difficult to perform; at times it is even impossible because sensor nodes are often positioned in inaccessible areas.

Various strategies have been addressed in order to reduce maintenance issues in measurement systems that are often disseminated. Some solutions, such as those implementing real time sensors, may require batteries; other systems, such as low duty cycle sensors and measurement architectures, may be able to function appropriately without batteries in specific working conditions [9]-[14].

Other solutions regard methods able to reduce or eliminate the power consumption during standby mode [15], [16].

In fact, several electronic parts need to be turned on only when an event occurs, being in standby the rest of the time with an associated power consumption.

It must be observed that during standby, most of the electronic parts are turned off except for the power management 
components that supply the microcontroller unit (MCU) and the front-end transmission, such as Bluetooth and RF [17].

For this reason, power management circuits are in fact permanently on in standby, and, depending on the duty cycle, can consume unnecessarily high percentages of battery charge [18], [19]. Standby leads to a significant savings, which can be translated into prolonged battery life or a reduction of battery size. This is beneficial in terms of cost and system miniaturisation [20].

With the aim of overcoming this problem, several solutions have been proposed in the literature regarding suitable design and circuit configuration [21].

A zero-standby solution, with discrete approach, has been presented by authors in order to remove the power dissipated during the standby of a measurement system, such as [15], [16] and [22].

Authors have also addressed the idea of using the zerostandby solution in the presence of MEMS; however, this approach has only been demonstrated through simulation with the aim of implementing an integrated chip [23]. In contrast to that study, the solution presented here involves more exhaustive simulations, the design of an integrated circuit and the experimental validation of the principle. It is worth noting that the proposed solution is suitable for MEMS and microscale transducers able to generate very weak voltage signals.

This study used a device based on PiezoMUMPs technology [24], which represents an interesting solution in order to realise self-generating integrated sensors.

This family of device can produce output voltages from tens to a few hundreds of $\mathrm{mV}$. It is worth noting that a more generalised study and characterisation (based on various voltage levels) of the designed and realised integrated conditioning circuit will be addressed in the experimental section, showing very intriguing features.

The paper is organised as follows: Section 2 describes the working principle. Section 3 reports the system description and simulations. Section 4 shows the setup and the results, Section 5 presents an analysis of the system, and the conclusions are given in Section 6.

\section{WORKING PRINCIPLE AND MEMS TECHNOLOGY}

This section addresses the idea of turning on a device that is completely off using only the energy provided by a MEMS device used as an energy transducer. The concept presented in this section goes beyond the standby functionality of a device, since it is intended to toggle the device from its off to its on state

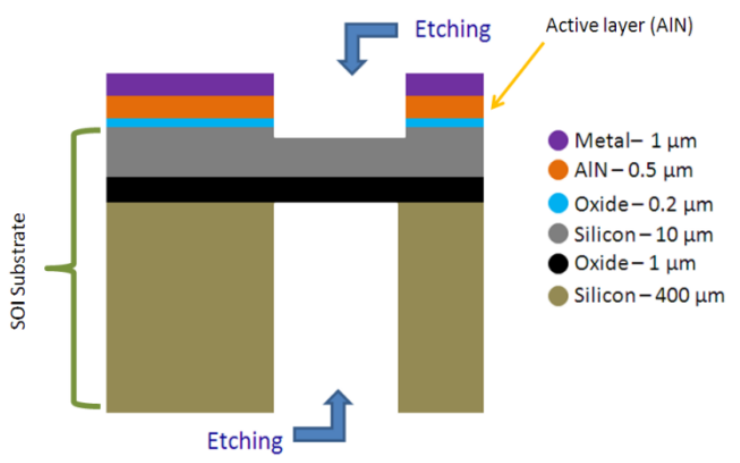

Figure 1. MEMS process used to realise integrated-scale sensors with output voltage thanks to the AIN layer.

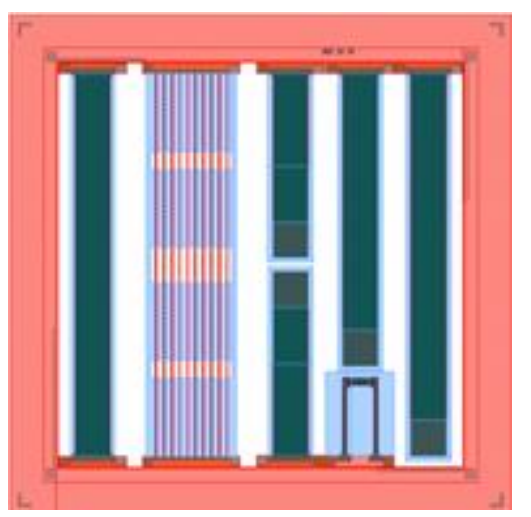

Figure 2. Layout of the MEMS, with several transducers in order to generate several output voltages.

without an intermediary standby state. A MEMS device is advantageous compared to other existing technologies, like PZT, due to the maturity and the wide diffusion of this technology. This inherently leads to low cost solutions and higher reliability. Furthermore, MEMS can also be adapted for sensing applications, creating an integrated system for cost optimisation. Thus, the aim is to use the MEMS device both as an energy transducer to turn on the application and as a sensor while the system is active. However, there are major challenges to achieving this. In fact, a MEMS device is normally used as a sensor rather than as an energy transducer due to its small size and energy density (in the order of few $\mathrm{nW}$ ). It also generates very low open circuit voltage, as low as $100 \mathrm{mV}$. These issues pose problems in the very early stage of the design. To tackle this, the MEMS must be designed in order to be energy efficient while also having an open circuit voltage high enough to trigger the system to turn on.

In this context, PiezoMUMPs technology [24] was used for the simulation and as a reference voltage level for the integrated circuit in the zero standby method. Figure 1 shows some details of the MEMS process.

As can be noted, the MEMS device was based on a silicon on insulator wafer, which was used as a substrate. The $10 \mu \mathrm{m}$ of silicon was a doped layer used to contact the AlN layer, having a thickness of $0.5 \mu \mathrm{m}$. An insulation layer of thermal oxide with a thickness of $200 \mathrm{~nm}$ separated the silicon layer and the AlN. The last layer was a metal stack composed of $200 \mathrm{~nm}$ chrome and $1,000 \mathrm{~nm}$ of aluminium. It was used as a second electrical contact for the AlN material. A deep reactive ion etching procedure was used to create a suspended structure. Figure 2 shows the layout of the MEMS, and Figure 3 shows the realised prototype that

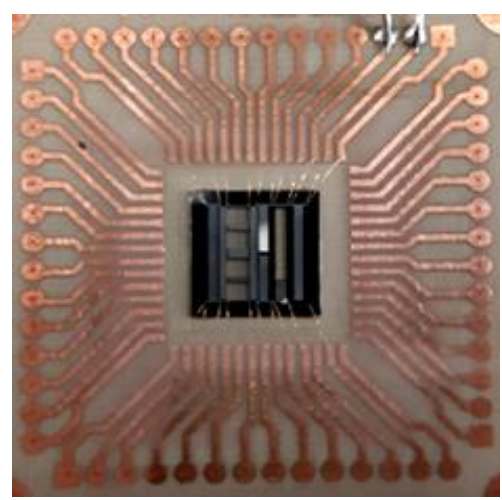

Figure 3. Picture of the realised bonded device. 
was used for simulations (see Section 3) and as reference voltage source for the experiments.

It is worth mentioning that the realised MEMS can generate a voltage output between $10 \mathrm{mV}$ and hundreds of $\mathrm{mV}$. Because of this, a suitable design for the conditioning circuit for the zero standby device must be addressed. This discussion will be presented with the design, realisation and characterisation in Section 4.

\section{SYSTEM DESCRIPTION AND SIMULATIONS}

Figure 4 represents a battery driven system such as a WSN that is composed of three main sections: 1) the vibration-based transducer, 2) a power management and 3) the final load, which in a typical application consists of sensors, micro controller units and generic transceivers (i.e. Bluetooth, RF, Wi-Fi, etc.).

The proposed vibration-based transducer for zero-energy standby applications is composed of two sections: (a) a MEMS device that works as a mechanical to electrical energy transducer able to convert external vibrations such as shocks and induced movements into electrical energy; and (b) a logic architecture able to efficiently manage power and convert the low voltage output of the MEMS device into level switching voltage. The idea is to supply the power requested by the electronic equipment only when the appliance is turned on by inducing kinetic energy through an impressed movement MEMS energy transducer that converts kinetic movement to an electric signal, $V_{\text {mens. }}$. When enough energy is provided to the MEMS harvester, it is able to connect the power path between the battery source, $V_{c c}$, and the output of the power management block, $V_{\text {out }}$. This, in turn, supplies a regulated voltage to the loading section. The logic section of the power management block does not have any static power consumption as long as the enable signal is low, and the leakage current flowing through the battery (few $\mathrm{pA}$ ) is, in practice, negligible. This block also ensures that the enabling signal of the voltage regulator can stay on even when the kinetic energy feeding the MEMS device has faded out.

Since the output voltage of a MEMS is very low $(\sim 100 \mathrm{mV})$, it would be insufficient to enable the voltage regulator, which normally requires a voltage as high as $V_{c c}$ to be turned on. For this reason, a special level shifter is needed to interface the MEMS-based transducer with the enable digital input of the voltage regulator.

The main purpose of the level shifter is to convert the small voltage provided by the MEMS device when moved by a mechanical force into a suitable logic level to enable the power management to bias the sensor node. In order to null standby-power consumption, it is fundamental that the circuit does not consume any power when the system is off.

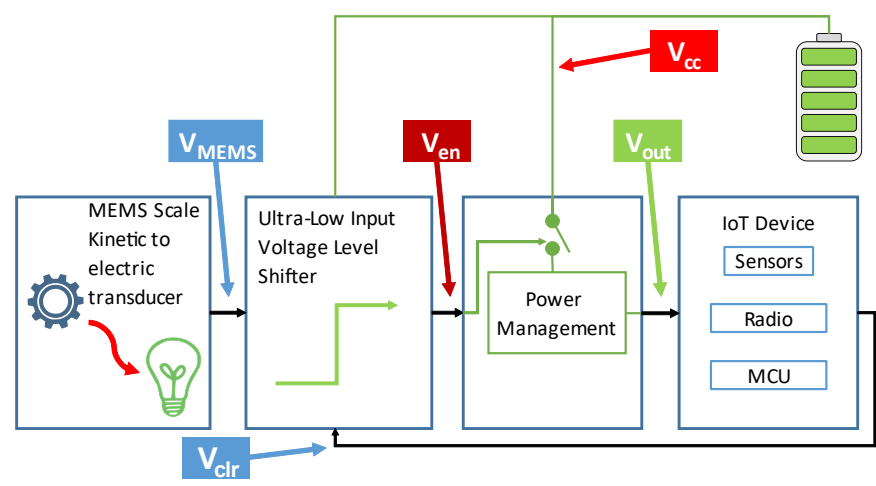

Figure 4. System block diagram.

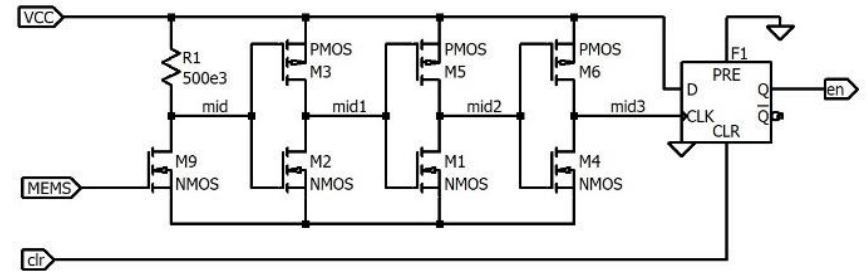

Figure 5. Schematic diagram of the proposed level shifter.

With reference to Figure 5, a visual inspection of the circuit shows that if the voltage $V_{\text {mems }}$ is zero, then transistor M9 is off. Also, the voltage $V_{m i d}$ will be high and equal to $V_{c c}$, accounting for the leakage in the transistor. Therefore, the inverter with transistors M2 and M3 is in a digital stable state with $V_{\text {mid }}$ set to zero and no current flowing through the transistors. The rest of the circuitry is digital, and thus does not consume any power, as shown in Figure 6a.

As shown in Figure 6b, the final flip-flop stays in its reset status so that the enable signal $V_{e n}$ is low and the power management is off; therefore, the rest of the system does not consume any power.

The working principle of the level shifter can be explained with reference to the simulated output as shown in Figure 7. Once displacement has been induced in the system, the MEMS device provides the signal $V_{\text {mems }}$ to the input at the gate of transistor M9. This, working sub-threshold, is able to pull down the voltage $V_{\text {mid }}$ when a small current flows through resistor R1. The following transistors will add gain to the signal $V_{\text {mid }}$ at each stage, so that the signal $V_{\text {mid }}$ will have sharp edges to latch the signal $V_{e n}$ through the D type flip-flop (F1).

Consider a stationary system where there is no displacement. Therefore, $V_{\text {mens }}=0$, and the current through resistor R1 is zero. After giving an external displacement to the system att $=550 \mu \mathrm{s}$, the $V_{\text {mens }}$ has a peak voltage that allows a small current to flow through the large resistor R1. This, in turn, provides a voltage

a)
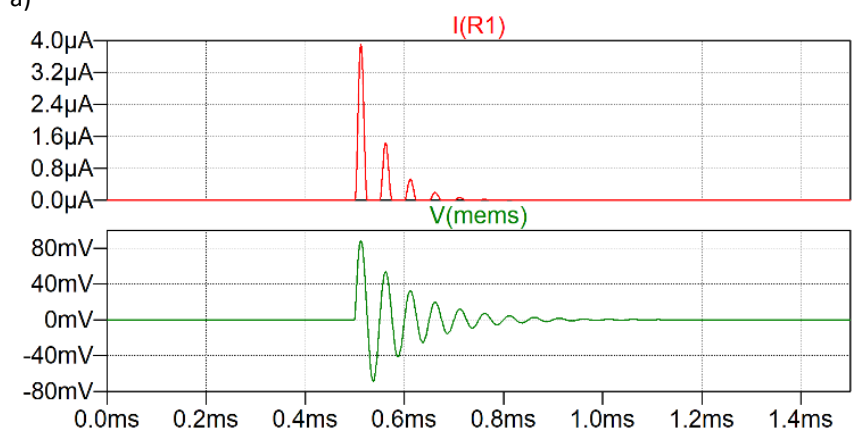

b)

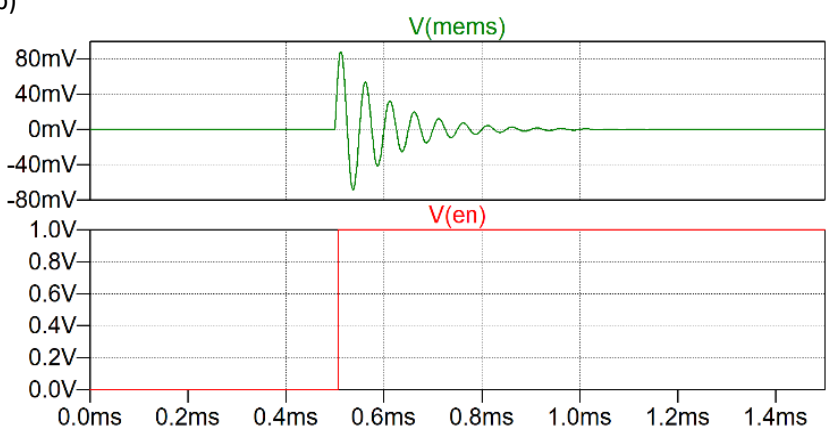

Figure 6. Simulation results of the circuit with respect to the system standby and active state. a) Current consumption during start up, b) Shift of enable signal from MEMS device. 


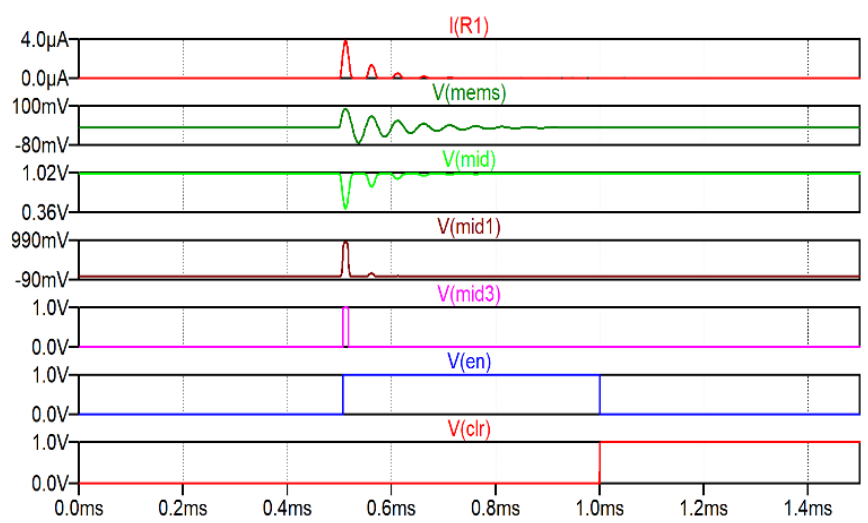

Figure 7. Analysis of the different node voltages in the circuit during turn-on sequence.

drop in the resistor of $2 \mathrm{~V}$ and brings $V_{\text {mid }}$ to $1 \mathrm{~V}$, as the bias across the branch is maintained by the supply at $3 \mathrm{~V}$. Since the voltage threshold of transistor inverters M2 and M3 is higher than $2 \mathrm{~V}$, the signal $V_{\text {mid }}$ gets inverted to $V_{\text {midt }}$. The following transistors will buffer the signal $V_{\text {midt }}$, providing the signal $V_{\text {mid }}$ that is used to latch the D type flip-flop for a high voltage output Q, so that the signal $V_{\text {en }}$ goes high.

It can be observed that once turned on, even if the system becomes stationery, the enable signal is maintained at $V_{c}$ irrespective of $V_{\text {mems. }}$. When needed, the flip-flop can be turned off by providing the high signal to $V_{c r}$ in order to reset it. This resets the signal $V_{\text {en }}$ to zero, which will turn off the system with no current consumption until the next impulse displacement is probed.

\section{INTEGRATED CIRCUIT AND EXPERIMENTS}

In order to support the simulation results, the proposed schematic was fabricated in the layout shown in Figure $8 \mathrm{a}$ into a die of dimensions $4 \mathrm{~mm} \times 4 \mathrm{~mm}$. A picture of the die is shown in Figure 8b. The die was then mounted on a PCB for external circuitry, as shown in Figure 8c. To characterise the circuit alone, an initial experimental setup was made to evaluate the triggering voltage of the level shifter with controlled DC voltages. This test setup consisted of a $3 \mathrm{~V}$ battery supply for $V_{c}$, powering the level shifter and a voltage divider circuit. The input of the circuit was fed by the voltage across the network of resistors varying from $52 \mathrm{mV}$ to $800 \mathrm{mV}$. The fabricated die was also covered by an opaque sleeve to reduce the leakage current due to light, and the circuit appeared to toggle at voltages as low as $52 \mathrm{mV}$. However, the required duration of the input was observed to be more than the simulation results, at an input voltage of $100 \mathrm{mV}$. It was also noticed that at slightly higher voltages $(\sim 270 \mathrm{mV})$ the toggle of the circuit was almost instantaneous, in the range of $\mathrm{ms}$. The results of the toggling speed at $92 \mathrm{mV}$ and $270 \mathrm{mV}$ are compared in Figure 9. Following the above observations, the integrated conditioning circuit was characterised based on the behaviour of the $V_{i n}$, and $V_{\text {out }}$ in terms of response time $\Delta t$ using the same setup. The results of this experiment are as summarised in Figure 10. It can be understood that the behaviour of the chip was as anticipated, but the resulting capacitance of the die was more than expected.

In the next section, the experimental study of the integrated circuit will be conducted, considering several voltage levels in accordance with the amplitude generated by the MEMS used in the simulation (see Figure 3) and the fabricated chip (see Figure 8). a)

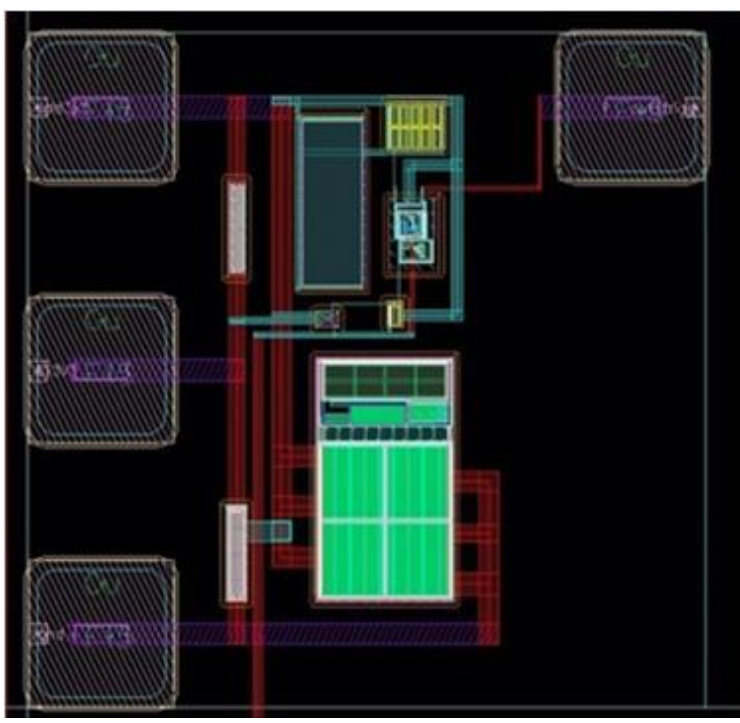

b)

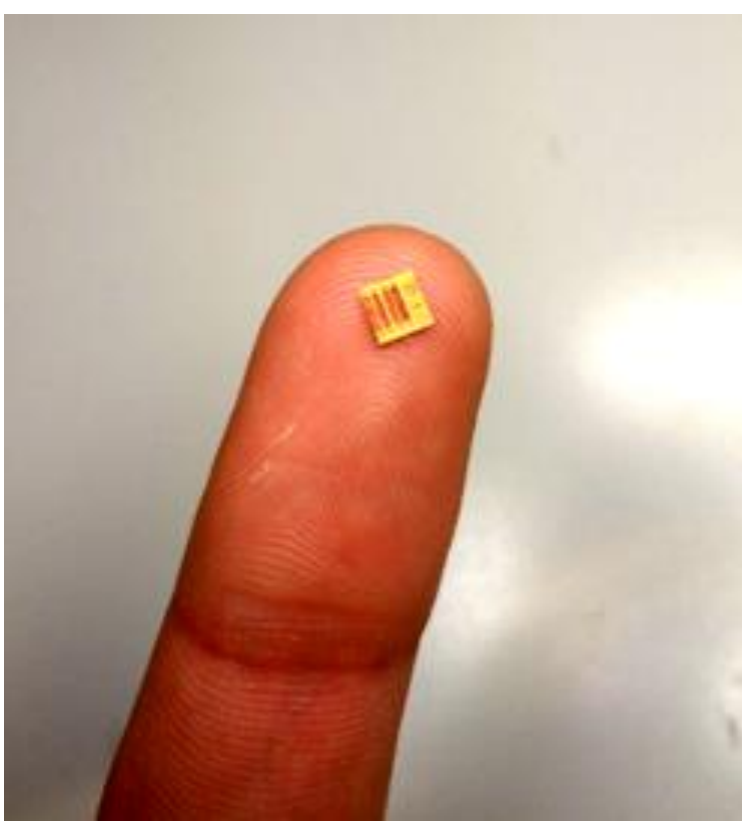

c)

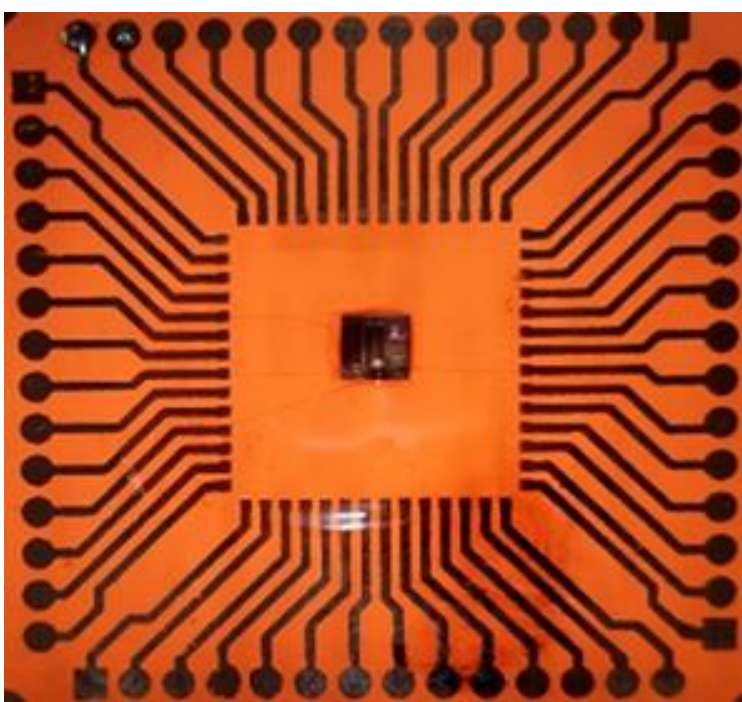

Figure 8. Integrated conditioning circuit for zero standby: a) layout view b) picture of the fabricated die, c) fabricated die mounted on a printed circuit board. 
a)

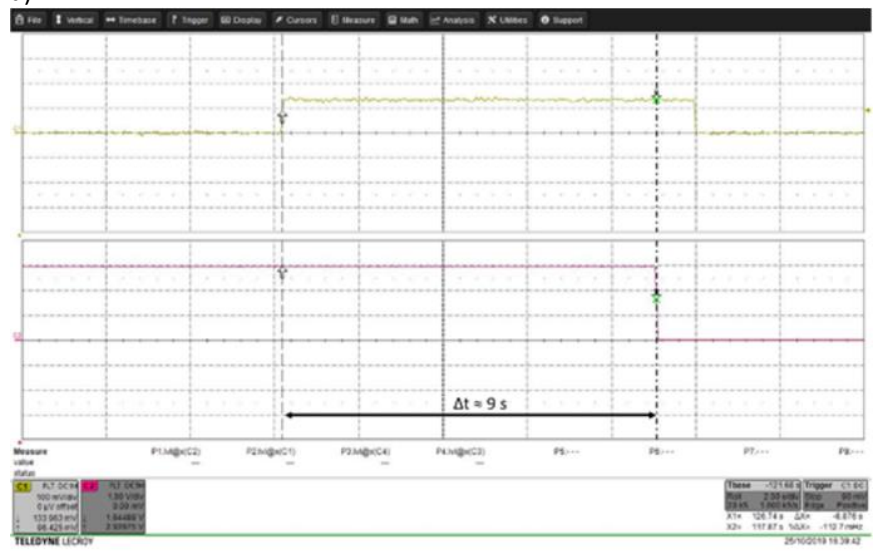

b)

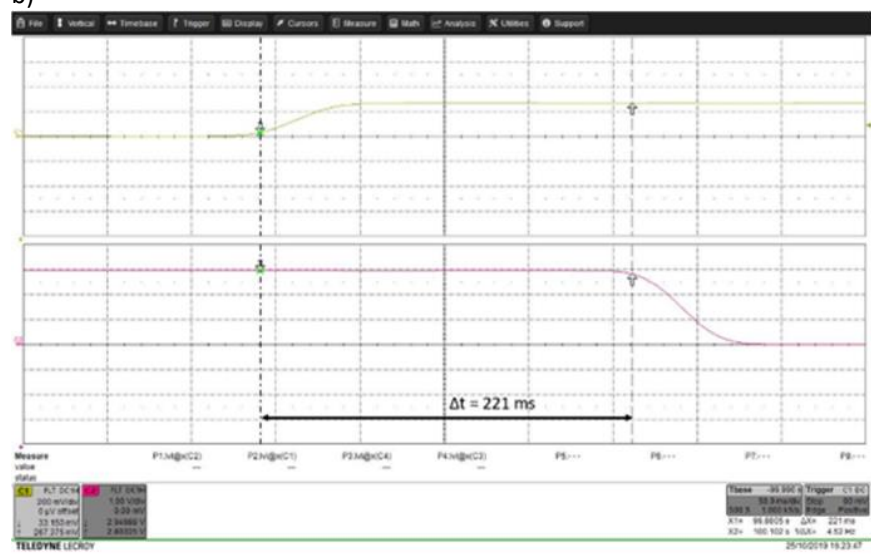

Figure 9. Results from the characterisation of the level shifter at various input voltages $\left(V_{\text {in }}\right):$ a) at $V_{\text {in }}=92 \mathrm{mV}$, the output toggles at $\Delta t=9 \mathrm{~s}$, b) at $V_{\text {in }}=270 \mathrm{mV}$, the output toggles at $\Delta t=221 \mathrm{~ms}$.

\section{SYSTEM ANALYSIS}

In order to study the chip for the zero standby device, an inverter (ST M74HC132B1) and a D type flip-flop (ST M74HC74B1) were connected to the setup as shown in Figure 11.

The board included the voltage divider branches, the die with the PCB, the inverter, the D type flip-flop and the battery.

Figure 12 shows the experimental setup used to characterise the system. In accordance with the simulation, the output Q of the flip-flop was set to high as long as the flip-flop was externally

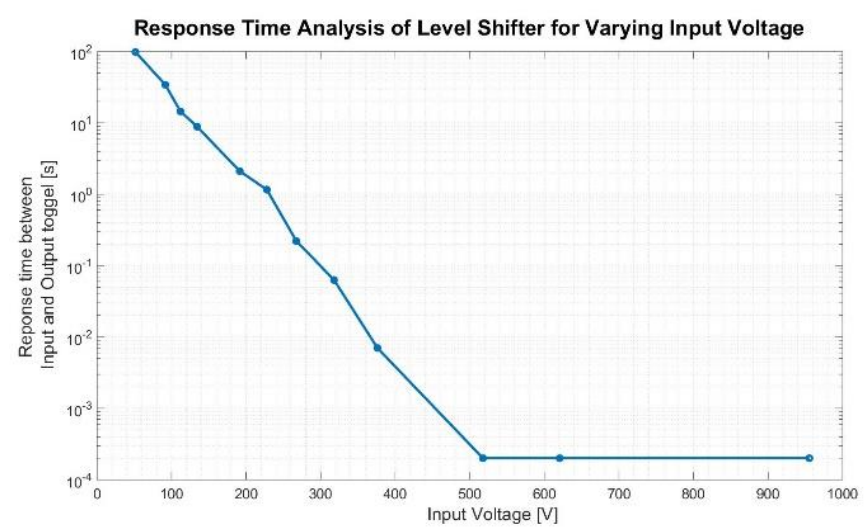

Figure 10. Response time analysis for the input and output voltages of the MEMS level shifter.

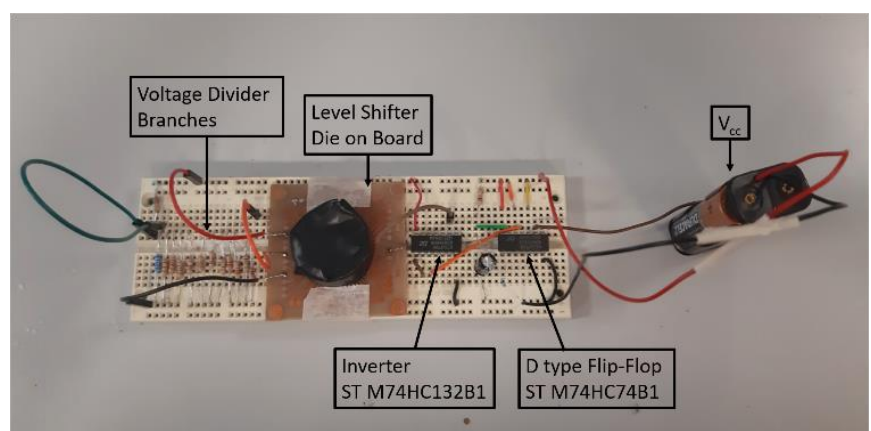

Figure 11. Experimental board combining the chip with ST M74HC132B1 inverter and ST M74HC74B1 D flip-flop.

reset through $V_{c l r}$. Figure 13 represents the different results obtained from the entire circuit shown in the set up. Clearly, there is a delay time and a need of constant input at very low voltages $(100 \mathrm{mV})$. But interestingly, the $V_{e n}$ does not toggle to digital high unless the $V_{d r}$ signal is given. This gives the user control over the active time of the sensor node/IoT device, allowing it to stay on until a $V_{c l r}$ is programmed from the MCU.

To accurately test and characterise the chip, the voltage divider bridge was replaced by an amplified piezoelectric actuator (APA). APA 50XS from Cedrat Technologies was used in this experiment.

The APA consisted of a rigid external shell used to produce stimulus at various voltage levels, casing a multi-layered piezoelectric stack. Therefore, when subjected to vibration, the kinetic force was translated into electrical voltage due to the piezoelectric effect, and the voltage was fed into the input terminal of the chip.

The APA was given a short mechanical impulse, and the response of the whole system at $V_{\text {in }}, V_{c l r}, V_{m i d z}$, and $V_{e n}$ was measured. The results are as shown in Figure 14, and they show very good agreement with the simulation results from the previous section.

\section{CONCLUSIONS}

In this paper, we addressed a zero-energy standby solution that is able to supply the power required by the measurement equipment to turn on an appliance only when needed. In particular, an integrated circuit (IC) solution suitable to be used with MEMS scale transducers was pursued. Both the MEMS transducer and the integrated conditioning circuit were

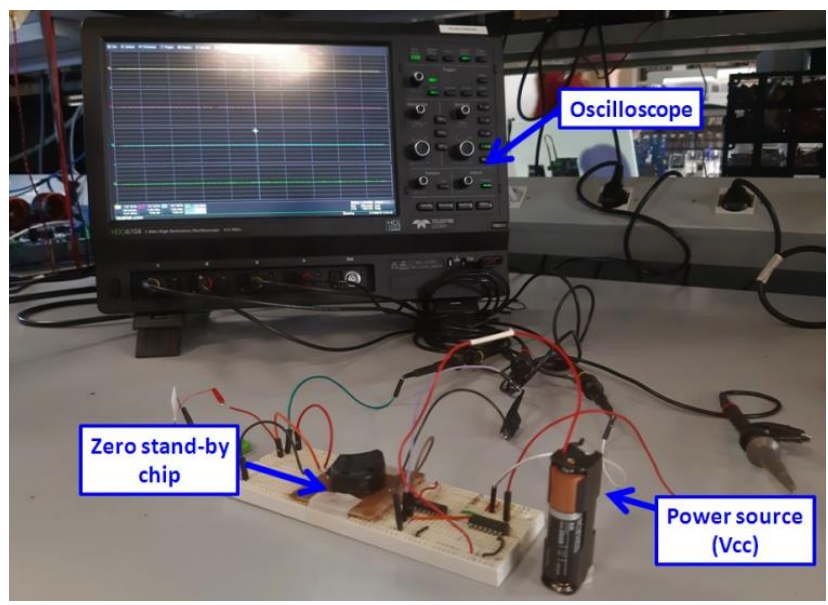

Figure 12. Experimental setup. 
a)

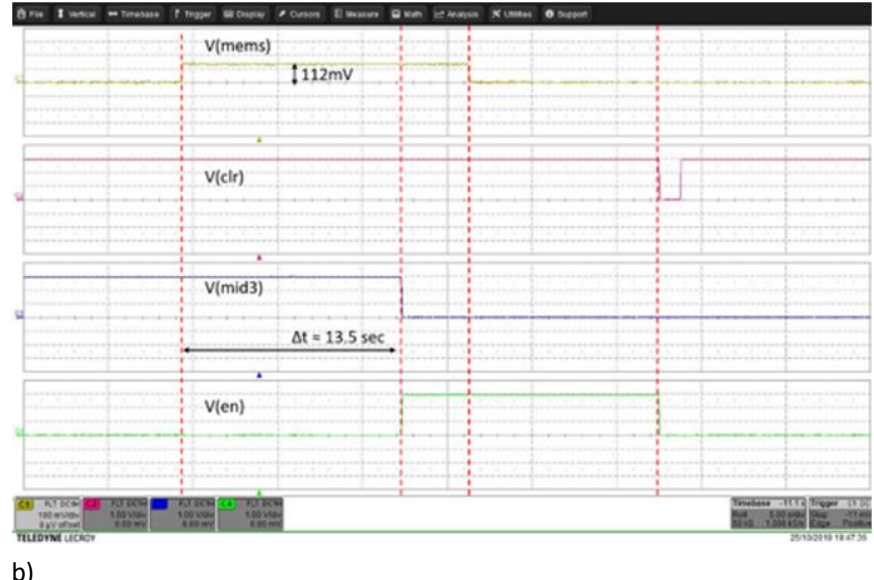

b)

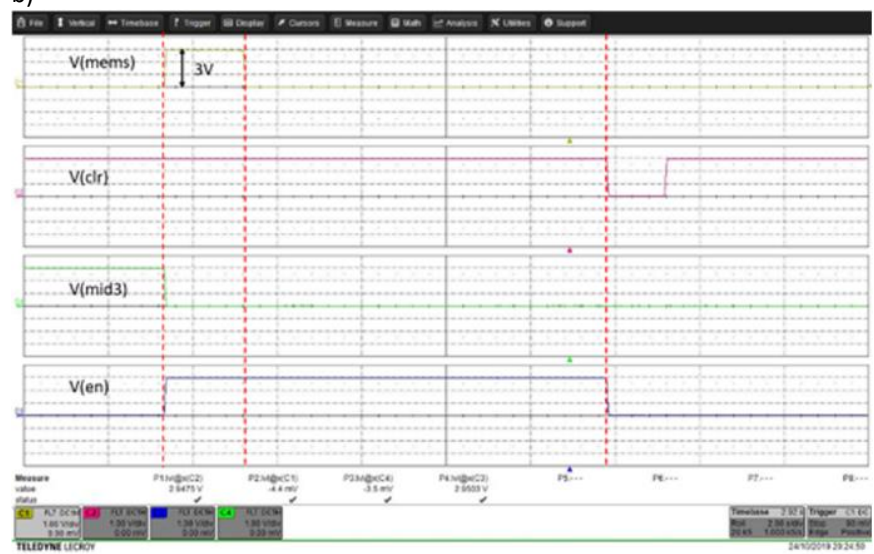

Figure 13. Results of the level shifter circuit for various input voltages: a) $V_{\text {in }}=112 \mathrm{mV}$, b) $V_{\text {in }}=3 \mathrm{~V}$

simulated, designed, realised and characterised, so that the zero-energy standby solution has been experimentally studied by using the realised devices. The IC is capable of working as an integrated level shifter with a very low threshold voltage $(<100 \mathrm{mV})$. This is advantageous and easily compatible with the majority of MEMS devices, as this value of voltage can be easily produced by several MEMS technologies. In this specific case, we chose the most commonly used MUMPs process, called PiezoMUMPs. Moreover, the tests showed that the performance obtained positively confirmed the purpose of the feasibility study. In addition to validating the methodology and the architecture of the system, the experiment provided relevant ideas for future improvements and optimisation for the

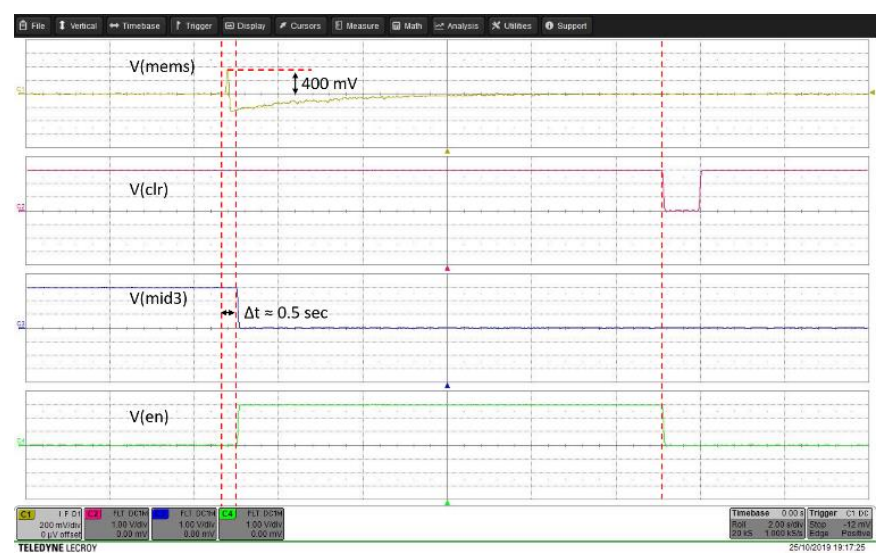

Figure 14. Experimental results from the combined circuit performance of both the MEMS and the integrated conditioning circuit. In particular, it should be noted that the level shifter requires minor improvements in terms of switching times, whereas for MEMS it is necessary to increase the open circuit voltage for low mechanical stresses. Continued study is in progress with a new design of both silicon-based devices in flip chip configuration in order to implement small scale measurement systems based on a zero-energy standby solution.

\section{REFERENCES}

[1] A. Burdett, Ultra-low-power wireless systems: Energy-efficient radios for the Internet of Things, IEEE Solid-State Circuits Magazine 7 no. 2 (2015), pp. 18-28.

DOI: https://doi.org/10.1109/MSSC.2015.2417095

[2] S. Park, S. W. Heo, W. Lee, D. Inoue, Z. Jiang, K. Yu, K. Fukuda, Self-powered ultra-flexible electronics via nano-grating-patterned organic photovoltaics. Nature 561 no.7724 (2018), pp. 516-521. DOI: https://doi.org/10.1038/s41586-018-0536-x

[3] C. Trigona, N. Dumas, L. Latorre, B. Andò, S. Baglio, P. Nouet, Exploiting benefits of a periodically-forced nonlinear oscillator for energy harvesting from ambient vibrations, Procedia Engineering 25 (2011), pp. 819-822.

DOI: https://doi.org/10.1016/i.proeng.2011.12.201

[4] F. Giusa, F. Maiorca, A. Noto, C. Trigona, B. Andò, S. Baglio, A diode-less mechanical voltage multiplier: A novel transducer for vibration energy harvesting, Sensors and Actuators A: Physical 212 (2014), pp. 34-41.

DOI: https://doi.org/10.1016/j.sna.2014.03.017

[5] C. Bachmann, M. Ashouei, V. Pop, M. Vidojkovic, H. De Groot, B. Gyselinckx, Low-power wireless sensor nodes for ubiquitous long-term biomedical signal monitoring, IEEE Communications Magazine 50 no. 1 (2012), pp. 20-27.

DOI: https://doi.org/10.1109/MCOM.2012.6122528

[6] A. Somov, E. F. Karpov, E. Karpova, A. Suchkov, S. Mironov, A. Karelin, D. Spirjakin, Compact low power wireless gas sensor node with thermo compensation for ubiquitous deployment. IEEE Transactions on Industrial Informatics 11 no. 6 (2015), pp. 1660-1670.

DOI: https://doi.org/10.1109/TII.2015.2423155

[7] M. Cerchecci, F. Luti, A. Mecocci, S. Parrino, G. Peruzzi, A. Pozzebon, A low power IoT sensor node architecture for waste management within smart cities context. Sensors 18 no. 4 (2018) 1282, 27 pages.

DOI: https://doi.org/10.3390/s18041282

[8] K. K. Patel, S. M. Patel, Internet of things-IOT: Definition, characteristics, architecture, enabling technologies, application \& future challenges, International Journal of Engineering Science and Computing 6 no. 5 (2016), pp. 6122-6131.

DOI: https://doi.org/10.4010/2016.1482

[9] A. R. Al-Ali, I. Zualkernan, F. Aloul, A mobile GPRS-sensors array for air pollution monitoring, IEEE Sensors Journal 10 no. 10 (2010), pp. 1666-1671. DOI: https://doi.org/10.1109/ISEN.2010.2045890

[10] R. J. Vullers, R. Van Schaijk, H. J. Visser, J. Penders, C. Van Hoof, Energy harvesting for autonomous wireless sensor networks, IEEE Solid-State Circuits Magazine 2 no. 2 (2010), pp. 29-38.

DOI: https://doi.org/10.1109/MSSC.2010.936667

[11] M. Zhu, M. Hassanalieragh, Z. Chen, A. Fahad, K. Shen, T. Soyata, Energy-aware sensing in data-intensive field systems using supercapacitor energy buffer, IEEE Sensors Journal 18 no. 8 (2018), pp. 3372-3383.

DOI: https://doi.org/10.1109/JSEN.2018.2809683

[12] B. Lyu, Z. Yang, G. Gui, Y. Feng, Wireless powered communication networks assisted by backscatter communication, IEEE Access 5 (2017), pp. 7254-7262.

DOI: https://doi.org/10.1109/ACCESS.2017.2677521 
[13] T. Soyata, L. Copeland, W. Heinzelman, RF energy harvesting for embedded systems: A survey of tradeoffs and methodology, IEEE Circuits and Systems Magazine 16 no. 1 (2016), pp. 22-57. DOI: https://doi.org/10.1109/MCAS.2015.2510198

[14] R. La Rosa, C. Trigona, G. Zoppi, C. Di Carlo, L. Di Donato, G. Sorbello, RF energy scavenger for battery-free wireless sensor nodes, Proc. of IEEE International Instrumentation and Measurement Technology Conference (I2MTC), Houston, USA, 14-17 May 2018, pp. 1-5. DOI: https://doi.org/10.1109/I2MTC.2018.8409738

[15] C. Trigona, B. Andò, S. Baglio, R. La Rosa, G. Zoppi, Vibrationbased transducer for zero-energy standby applications, Proc. of IEEE Sensors Applications Symposium (SAS), Catania, Italy, 2022 April 2016, pp. 1-4.

DOI: https://doi.org/10.1109/SAS.2016.7479817

[16] C. Trigona, B. Andò, S. Baglio, R. La Rosa, G. Zoppi, Sensors for kinetic energy measurement operating on "zero-current standby", IEEE Transactions on Instrumentation and Measurement 66 no. 4 (2017), pp. 812-820.

DOI: https://doi.org/10.1109/TIM.2016.2644838

[17] Y. Zhang, L. Qun, Exploiting Zigbee in reducing WIFI power consumption for mobile devices, IEEE Transactions on Mobile Computing 13 (2014), pp. 2806-2819.

DOI: https://doi.org/10.1109/TMC.2014.2315788

[18] Y. Rao, D. P. Arnold, An input-powered vibrational energy harvesting interface circuit with zero standby power, IEEE Transactions on Power Electronics 26 (2011), pp. 3524-3533. DOI: https://doi.org/10.1109/TPEL.2011.2162530

[19] A. Yamawaki, S. Serikawa, Battery life estimation of sensor node with zero standby power consumption, Proc. of IEEE International Conference on Computational Science and
Engineering (CSE) and IEEE Intl Conference on Embedded and Ubiquitous Computing (EUC) and $15^{\text {th }}$ Symposium on Distributed Computing and Applications for Business Engineering (DCABES), Paris, France, 24-26 August 2016, pp. 166-172.

DOI: https://doi.org/10.1109/CSE-EUC-DCABES.2016.179

[20] R. La Rosa, N. Aiello, G. Zoppi, RF remotely-powered integrated system to nullify standby power consumption in electrical appliances, Proc. of IECON 2016, 42 $2^{\text {nd }}$ Annual Conference of the IEEE Industrial Electronics Society, Florence, Italy, 24-27 October 2016, pp. 1162-1164. DOI: https://doi.org/10.1109/IECON.2016.7793079

[21] K. H. Jones, J. Gross, Reducing size, weight, and power (SWaP) of perception systems in small autonomous aerial systems, Proc. of $14^{\text {th }}$ AIAA Aviation Technology, Integration and Operations Conference, Atlanta, USA, 16-20 June 2014, AIAA 2014-2705, pp. 1404-1412.

DOI: https://doi.org/https://doi.org/10.2514/6.2014-2705

[22] R. La Rosa, P. Livreri, C. Trigona, L. Di Donato, G. Sorbello, Strategies and techniques for powering wireless sensor nodes through energy harvesting and wireless power transfer. Sensors 19 no. 12 (2019) 2660. DOI: https://doi.org/10.3390/s19122660

[23] R. La Rosa, C. Trigona, B. Andò, S. Baglio, MEMS based transducer for zero-energy standby application, Proc. of II Workshop on Metrology for Industry 4.0 and IoT (MetroInd4. 0\&IoT), Naples, Italy, 4-6 June 2019, pp. 12-15. DOI: https://doi.org/10.1109/METROI4.2019.8792888

[24] Memscap, PiezoMUMPs. Online [Accessed 30 October 2020] http://www.memscap.com/products/mumps/piezomumps 